\title{
Dissecção de Artérias Cervicocefálicas
}

\author{
S. E. de Melo-Souza*, C. N. Raffin*
}

\section{RESUMO}

Identificação de fatores de risco nas doenças vasculares encefálicas (DVE) tem contribuido na diminuição de sua incidência. Dois grupos de sintomas foram identificados: o primeiro decorre do fenomeno local e o segundo devido à alteraçăo na circulaçāo cerebral pela conseqũente isquemia hemorrágica. A principal característica á a dor na região da dissecçảo e que, geralmente, irradia pelo trajeto distal até a artéria comprometida. Na casuística foram avaliados 161 pacientes (135 da artéria carótida Interna e 26 vertebral), $68 \%$ exibiram cefaléia com dissecção da artéria carótida interna e $69 \%$ da artéria vertebral, tendo sido a manifestaçāo inkcial de $47 \%$, quando envolve a artéria carótida interna e $33 \%$ envolvendo a artéria vertebral. Os autores demonstraram os recentes avanços em DVE e suas experiéncias de diagnóstico $\theta$ tratamento.

\section{UNITERMOS}

Doenças vasculares encefálicas, dissecçāo

\footnotetext{
- Neurologistas do Instituto de Neurologia de Goiânia
}

\section{INTRODUÇÃO}

As doenças vasculares encefálicas (DVE) continuam sendo uma das mais frequientes e sérias patologias, a terceira causa de mortalidade e a primeira em desabilidades. Nos Estados Unidos há 500000 casos novos com 150000 mortes a cada ano. Uma grande porcentagem dos que sobrevivem tem seqüelas de maior ou menor monta que podem exigir cuidados especiais de familiares, com grande custo econômico.

Em nosso país, presume-se à base de alguns relatos e na experiência pessoal que doenças infectoparasitárias, como a doença de Chagas e a moléstia reumática, possam acrescentar mais casos de DVE e possivelmente atingir populações menos idosas.

A identificação de fatores de risco capazes de influir fortemente na incidência das DVE tem trazido grandes benefícios pela viabilidade de, através de seu manuseio, alterar a história natural das DVE, diminuindo a sua incidência. Hipertensão arterial, tabagismo, alterações eletrocardiográficas, diabetes mellitus, dislipidemias, alcoolismo, anticoncepcionais orais acima dos 30 anos podem ser corrigidos, refletindo nitidamente no curso das doenças vasculares em geral e nas encefálicas em especial.

Recentemente tem sido chamada a atenção para um mecanismo etiopatogênico que foi claramente subdiagnosticado até então. Trata-se do processo de dissecção da parede arterial, consistindo na penetração de sangue na parede arterial.

A dissecção vascular cervical espontânea ocorre predominantemente em adultos jovens e pessoas de meia-idade, sendo rara em adolescentes e crianças. As dissecçōes arteriais intracranianas, principalmente da circulação anterior, são vistas em grupos mais jovens, com metade dos casos nas primeiras duas décadas de vida ${ }^{28}$. A relação entre dissecações arteriais extracranianas do território anterior e posterior é semelhante entre adultos e crianças, embora alguns autores relacionem que as dissecções intracranianas em adultos são mais comumente vistas no território posterior, enquanto em crianças são mais frequientes no território anterior ${ }^{28}$. 
Parecem predominar nas mulheres as dissecções da artéria vertebral extracraniana, usualmente bilateral, com reversão mais usual que nos homens, em que predomina dissecção da artéria vertebral intracraniana, usualmente unilateral e com extensão para a artéria basilar, com prognóstico mais reservado .

Inicialmente creditada apenas ao traumatismo óbvio nos vasos do pescoço ou por extensão da aorta, passouse a admiti-la também em traumas menores, leves e triviais, como na rotação brusca ou hiperextensão do pescoço, exercícios, fenômeno da ponta do chicote, prática de esportes e manipulação quiroprática. A dissecção espontânea, especialmente após MillerFischer ${ }^{9}$, foi primeiramente descrita para a artéria carótida interna extracraniana, depois para a vertebral cervical, em seguida para as artérias intracranianas de circulação posterior e finalmente para os vasos intracranianos oriundos das carótidas. Atualmente é considerada como importante mecanismo na etiologia das DVE. A incidência anual de dissecção da artéria carótida interna espontânea é de 2,6 para 100.000 pessoas $^{24}$. Atualmente reconhecida como causa importante de DVE especialmente no grupo mais jovem, presumindo-se em 0,4 a $2,5 \%$ dos casos de DVE na população em geral e de 5 a $20 \%$ nos mais jovens ${ }^{25}$, e $2 \%$ das doenças da artéria carótida interna ${ }^{27}$.

A dissecção consiste na ruptura da íntima, que pode ser facilitada por patologias preexistentes, como doenças do tecido conjuntivo, síndrome de Marfan, EhlersDanlos, displasia fibromuscular, doença de Behçet, hipertensão arterial e enxaqueca. Descreve-se em alguns casos a necrose cística da túnica média. A redundância da carótida parece ser um fator de risco'. A posição em hiperextensão prolongada do pescoço durante a anestesia geral pode desencadeá-la". Foi descrita em casos de afibrinoginemia ${ }^{10}$, de deficiência de alfa 1 -antitripsina ${ }^{29}$ e em paciente com substituição da alanina por glicina (G13A) na cadeia alfa 1 do colágeno tipo $I^{21}$, o que demonstra a pesquisa insistente em se conhecer por que ocorre a dissecção espontânea das artérias. No momento atual não se determina o motivo da dissecção na maioria das vezes. Não há preferência por sexo ou raça e a idade é muito variável (casos de 3 a 80 anos), com idade mediana de 42 anos, mas parece predominar nos indivíduos jovens ${ }^{25}$, correspondendo a $6,8 \%$ das causas de DVE abaixo dos 18 anos de idade e compromete tanto as artérias carótidas como as vertebrais ${ }^{27}$.

Nas artérias extracranianas, a penetração do sangue disseca a parede em sentido longitudinal, ao longo da camada média. $\mathrm{O}$ sangue pode retornar à luz do vaso rompendo a íntima, ou então, atinge a adventícia, provocando uma dilatação assimétrica da artéria. $O$ mais comum é que o hematoma apenas reduza a luz arterial, mas pode levar à oclusão total. Do local de estenose, podem-se desprender êmbolos para a circulação intracraniana $^{22}$.

Enquanto o pleno de dissecção das artérias extracranianas carótidas ou vertebrais é dentro da média, nas artérias intracranianas a média é significativamente simplificada e o plano de dissecção é geralmente subintimal e pode se estender através da adventícia ${ }^{28,37}$.

As artérias intracranianas, mais frágeis por não possuírem camada elástica externa, podem permitir o extravasamento imediato de sangue, ou formar um aneurisma que pode se romper posteriormente, levando a hemorragia subaracnoídea ou intraparenquimatosa. Deste modo, a dissecção pode determinar tanto um quadro isquêmico ou hemorrágico, ou de um tipo sucedendo ao outro.

As artérias carótidas internas extracranianas são as demais reconhecidas vítimas da dissecção, o que geralmente ocorre a dois ou mais centímetros de sua origem. As vertebrais podem ser afetadas em quaisquer segmentos, mas principalmente em sua parte média ou distal ${ }^{2}$. Os vasos intracranianos parecem ser menos atingidos que os extracranianos, mas talvez por ser de mais difícil comprovação, os da circulação posterior são mais relatados $6,7,12,13,14$.

\section{QUADRO CLINICO}

Há dois grupos de sintomas e sinais: o primeiro decorre do fenômeno local da dissecção e o segundo devido à alteração na circulação cerebral pela consequiente isquemia ou hemorragia ${ }^{22}$.

A principal característica da dissecção é a dor na região da dissecção e que geralmente se irradia pelo trajeto distal da artéria comprometida $(22,30,33$ a). De 161 pacientes avaliados ( $135 \mathrm{da}$ artéria carótida interna e 26 da vertebral), $68 \%$ exibiram cefaléia com dissecção da artéria carótida interna e $69 \%$ da artéria vertebral, tendo sido a manifestação inicial em $47 \%$ quando envolve a artéria carótida interna e $33 \%$ se a artéria vertebral $^{30}$. A dor pode preceder de horas a poucos dias os sintomas neurológicos e é sempre do lado da dissecção. Em dissecções bilaterais do sistema vertebrovasilar surpreendentemente a dor costuma ser unilateral $^{6}$. Na carótida, a dor é na face lateral do pescoço, região parotídea, ramo ascendente da mandíbula e região fronto-temporal, enquanto na vertebral, a dor é na face posterior do pescoço e região occipital, às vezes com posição antálgica e bloqueio. A dor é quase sempre firme, poucas vezes pulsátil, de 
média a forte intensidade e costuma durar de alguns dias até uma ou duas semanas $(30,33$ a). A dor com estas características passa a ser um sintoma de alarme e é de valor diagnóstico em casos de DVE (33 a).

DVE devido a dissecção arterial foi fortemente associada com cefaléia, enquanto DVE por embolismo (cardíaco ou artério-arterial) foi mais freqüente sem cefaléja, enfatizando o papel dos vasos extra e intracranianos na gênese da cefaléia no início do acidente vascular ${ }^{16}$.

$\mathrm{Na}$ dissecção da artéria carótida interna pode haver comprometimento do plexo simpático paricarotídeo e manifestar uma síndrome de Horner ipsilateral. É importante lembrar que nestes casos a sudorese da face está preservada porque as fibras simpáticas para a inervação das glândulas sudoríparas percorrem seu trajeto em íntimo contato com a artéria carótida externa. Pode-se desenvolver um sopro que é audível até à distância. Aumento de volume da carótida não é comum, mas pode haver dolorimento à palpação local (carotidinia). Pode haver comprometimento de nervos cranianos vizinhos com manifestações clínicas específicas, tais como dor facial, dor na região amigdaliana (IX nervo), disfonia e diafagia (X nervo) e desvio da linguagem (XII nervo) ${ }^{12}$ talvez por interferência em sua vascularização.

Quando a artéria vertebral é comprometida, além da dor, da posição antálgica e do bloqueio cervical, pode haver déficit motor de tipo periférico no membro superior ipsilateral ${ }^{8}$. As dissecções da artéria vertebral extracraniana são muito mais freqüentes que da parte intracraniana desta artéria, e estas costumam ser mais comumente unilaterais ${ }^{6}$. Os achados patológicos observados na porção intracraniana são diferentes daqueles da região extracraniana por causa das diferenças de anatomia vascular e morfologia. As artérias modificam-se ao penetrarem na região intracraniana, de modo que a muscular e a adventícia são cerca de dois terços das artérias extracranianas, acrescentandose que não possuem uma camada elástica externa bem desenvolvida ${ }^{6}$. A dissecção pode-se resumir a estes sintomas locais e o quadro esvanecer-se em poucos dias, porém há raros casos de dissecção crônica em estágios diferentes da evolução. ${ }^{26,37}$

As manifestações vasculares encefálicas isquêmicas podem ser discretas, transitórias ou levar a infartos de extensão variável com conseqüências por vezes graves ${ }^{4}$. A síndrome de Wallenberg incompleta é reconhecida como causada pela dissecção da artéria vertebral em cerca de $80 \%$ dos casos ${ }^{13}$. Mais frequientemente, dor ou disestesia no olho ou face ipsilateral e vertigem são referidas. Vômitos, visão borrada, diplopia e oscilos- copia são também comuns nas dissecções da artéria vertebral extracraniana. Em alguns casos, síndrome cerebelar pode ser observada, porém o tremor de intenção está raramente presente.

Hemorragia cerebral e principalmente subaracnóidea podem ser produzidas por dissecção de artérias intracranianas ou por extensão das artérias extracranianas, o que parece ocorrer mais freqüentemente em algumas horas ou dias após a dissecção, porém descrição de sinais e sintomas iniciando-se duas semanas até seis meses são descritos na literatura ${ }^{6}$.

A real incidência de dissecção arterial dos vasos intracranianos na gênese das DVE ainda está por ser estabelecida e acredita-se que o quadro possa ter curso menos benigno que o extracraniano. Na literatura há mais casos descritos no sistema vertebrobasilar que no carotídeo ${ }^{6.7 .13,14}$. Além disto, é importante enfatizar que a dissecção das artérias intracranianas pode ocasionar sintomas isquêmicos, com ou sem a formação de aneurisma, ou hemorragia parenquimatosa ou subaracnóidea na fase aguda ou mais tardiamente ${ }^{7}$.

\section{DIAGNÓSTICO}

À suposição clínica, deve-se proceder a exames complementares que possam corroborar o diagnóstico. $\mathrm{O}$ método definitivo é a angiografia cerebral convencional, por cateterismo arterial, idealmente com a técnica da subtração óssea (angiografia digital). Demonstra-se assim o local da dissecção, a presença ou não de estenose ou aneurisma, o falso e o duplo lúmen, além de avaliar a circulação colateral ${ }^{9,22}$.

Devido aos riscos na execução do cateterismo, há bastante ênfase na busca de métodos não invasivos que possam levar ao diagnóstico com segurança. Os procedimentos que têm evoluído enormemente são a ultrassonografia, a angiografia por tomografia computadorizada helicoidal e a angiografia por ressonância magnética. Todos estes métodos têm validade e parecem equivaler-se na sensibilidade, embora a especificidade não seja tão elevada. Estes métodos também são os mais práticos, com facilidade de repetição para o acompanhamento evolutivo das lesões. Ressalve-se que a tomografia helicoidal requer grande carga de contraste iodado, a ultrassonografia comum não avalia os vasos intracranianos e a ressonância magnética é o exame mais dispendioso de todos estes.

A ultrassonografia é capaz de demonstrar estenose ou obstrução arterial da carotida e pode sugerir que se trate de dissecção quando existe um afinamento progressivo do vaso, pela presença de uma membrana 
na luz ou se houver imagem de duplo lúmen. São também bastantes sugestivos a presença de focos ecogênicos intra-luminais associado à freqüência sistólica reduzida e fluxos em direção alternante ${ }^{32}$. As vertebrais também podem ser identificadas e avaliadas desde a sua origem até a porção intermediária. Os ultrassons coloridos têm algumas vantagens que facilitam o diagnóstico.

Em 14 casos de dissecção vertebral espontânea, não foi encontrado achado patognomônico pelo ultrassom. Contudo, se o paciente tinha sinais e sintomas sugestivos, o exame pode corroborar a suspeita clínica e ajudar na decisão de anticoagulação precoce.

O mapeamento de fluxo a cores é um método confiável de diagnóstico precoce na dissecção da artéria vertebral extracraniana ${ }^{2}$. Espessamento de parede, estenose ou oclusão da luz, falso lúmen e aneurisma podem ser identificados mesmo na fase inicial.

O doppler transcraniano vasculha a circulação intracraniana e pode auxiliar na avaliação global da circulação; também pode detectar microembolias partindo dos vasos dissecados ${ }^{31}$.

A tomografia computadorizada helicoidal é também um bom método de diagnóstico de dissecção de vasos extracranianos, estudando-os longitudinalmente ou em cortes transversais ${ }^{18}$. É possível demonstrar a luz residual, hematoma de parede e aneurismas. O lúmen excêntrico é específico. O "sinal do alvo', caracterizado como uma fina camada de contraste em torno da luz do vaso, também é altamente sugestivo, embora não muito freqüente. A tomografia computadorizada convencional ou não helicoidal também pode demonstrar as alterações em cortes transversais, mas tem pouca sensibilidade.

A ressonância magnética pode visualizar artérias em cortes transversais e identificar lesões ${ }^{25,37}$. Os achados sugestivos à ressonância são: (a) aumento do sinal do vaso inteiro, (b) uma borda de aumento de sinal em torno do lúmen, com estreitamento da luz vascular, (c) pobreza ou ausência de visualização do vaso, e (d) significativo comprometimento do lúmen vascular por um aumento anormal do sinal do tecido adjacente ${ }^{35}$. No diagnóstico diferencial de dissecção vascular pela ressonância magnética devem ser considerados os artefatos de fluxo pelos ateromas, coágulos intraluminares e artefato de registro devido ao fluxo oblíquo do eixo do gradiente de codificação de fase ${ }^{17}$. Um diagnóstico definitivo pode ser feito de modo não invasivo quando a ressonância magnética demonstra hematoma na parede do vaso, constituindo um dos achados mais significativos na neuroimagem desta patologia ${ }^{34}$.

Contudo, o estudo angiográfico pela ressonância magnética é mais abrangente e parece ter a mesma acuracidade da angiografia por tomografia helicoidal ${ }^{15}$, sendo bastante seguro nas dissecções da artéria carótida extracraniana, mas bem inferior à angiografia convencional do sistema vertebral ${ }^{19}$.

Em estudo de cinco pacientes com dissecção arterial carotídea utilizando a ressonância magnética, o diagnóstico de falso positivo foi feito em um paciente em que foi identificada turbulência e susceptibilidade magnética ao nível do canal petroso, no qual hiperplasia fibromuscular foi identificada pela angiografia convencional mas não pela angiografia pela ressonância magnética ${ }^{23}$.

\section{CONDUTA}

Sabendo-se que existem casos benignos, de resolução espontânea, sem complicações neurológicas, uma conduta expectante poderia ser indicada, principalmente para a maior parte das dissecções extracranianas. Quando as artérias extracranianas ocluem-se, mais frequientemente não se recanalizam. Entretanto se mantiverem algum lúmen a tendência é de normalizar a luz vascular. Já para as artérias intracranianas, a incidência de recanalização espontânea não é conhecida. Entretanto, a maioria dos autores introduz drogas antiagregantes plaquetários (ácido-acetilsalicílico ou ticlopidina) ou indica anticoagulação plena com heparina, seguida pelo uso de anticoagulantes orais. Isto justifica-se pela análise de quase todas as estatísticas publicadas ${ }^{3,45.20,33 \mathrm{~b}}$. O seguimento evolutivo pode ser feito de modo menos agressivo empregando-se a angiografia por ressonância magnética, tomografia computadorizada helicoidal ou ultrassonografia.

A abordagem cirúrgica direta ao nível da dissecção não é indicada, exceto na presença de aneurisma ${ }^{14}$. A oclusão por procedimento endovascular pode ser utilizada nos aneurismas persistentes ${ }^{36}$.

$O$ infarto cerebral costuma ocorrer nas primeiras horas ( $88 \%$ dos casos), mas pode ser mais tardio, nas semanas subsequientes $(12 \%)^{4}$. Isto pode orientar no sentido de se iniciar o tratamento o mais precocemente, mantê-lo no primeiro mês ou até no máximo o terceiro mês 5 .

\section{RECORRENCIA E EVOLUÇÃO}

Confirmando o que a experiência parecia demonstrar, a recorrência da dissecção na mesma artéria não é freqüente, ficando abaixo de $8 \%$ para acompanhamentos 
prolongados, com uma incidência média de $1 \%$ ao ano. $\mathrm{O}$ maior perigo reside no primeiro mês ${ }^{3,26,32}$. O uso continuado de antiagregantes plaquetários e de anticoagulantes não previne a recorrência ${ }^{3}$. Além disso, caso aconteça, a recorrência poderá ser benigna e sem sinais de comprometimento neurológico ${ }^{3,20}$. A cefaléia é o sintoma que costuma durar mais tempo (20\%), podendo inclusive ser seqüela da dissecção de artérias cervicais ${ }^{20}$. A recanalização da artéria acontece em $50 \%$ dos casos e existem cerca de $5 \%$ de possibilidades de formação de aneurisma ${ }^{20}$. A dissecção em outra artéria pode acontecer, inclusive dissecções múltiplas ${ }^{2,26}$. A história natural da dissecção arterial vertebral não rota parece relativamente benigna, com grande probabilidade de cura ${ }^{37}$. Alguns estudos retrospectivos de dissecção carotídea cervical, contudo, relatam um prognóstico menos favorável ${ }^{24}$.

\section{SUMMARY}

Identification of risk factors for encephalic vascular diseases (EVD) has contributed to reducing the incidence of this condition. Two groups of symptoms have been identified: those resulting from local phenomena and those which are caused by changes in brain circulation resulting from hemorrhage ischemia. Main characteristic is pain in the dissected artery area that radiates via the distal trajectory to compromised artery. In a study of cases evaluating 161 patients submitted to artery dissection (135 of the internal carotid artery and 26 of the vertebral carotid), $68 \%$ and $69 \%$ developed cephalea after dissection of the internal carotid artery and vertebral artery, respectively. This symptom had early manifestations in $47 \%$ of patients when the internal carotid artery was involved, and $33 \%$ when the vertebral artery was involved. The authors reported recent developments in EVD and their experience in the diagnosis and treatment of this condition.

\section{KEY WORDS}

Encephalic vascular diseases, dissection

\section{Referências}

1. BARBOUR, P.J.; CARTALDO, J.E.; ERA-GRANT, A.D.; GEE,W; REED, J.R. 3rd.; JENNI, D-P-; LONGENNECKER, J. - Internal carotid artery redundancy is significantly associated with dissection. Stroke 25: 1202-1206, 1994.

2. BARTELS, E.O.; FLUGEL, K.A. - Evaluation of extracranial vertebral artery dissection with dupplex clor-flow imaging. Stoke; 27: 290-295, 1996

3. BASSETTI, C.; CARRUZZO, A.; STURZENEGGER, M. \& TUNCDOGAN, E. - Recurrence of cervical artery dissection: a prospective study of 81 patients - Strolke, 27: 1804-1807, 1996.

4. BIOUSSE, W.; D'ANGLEJAN-CHATILLON, J.; TOUBOUL, P.J.; AMARENCO, P \& BOUSSER, M.G. - Time course of symptoms in extracranial carotid artery dissections; a series of 80 patients - Stroke, 26: 235-239, 1985.

5. CAPLAN, LR. - Stroke: a clinial aproach, 2nd edition, Butterworth-Heinemann, 1993, pags. 229-304.

6. CAPLAN, L.R. \& TETTENBORN, B. Vertebrobasilar occlusive disease: review of selected aspects. I. Spontaneous dissection of extracranial and intracranial posterior circulation arteries. Cerebrovasc Dis., 2: 256-265, 1992.
7. DINIZ-CARNEIRO, D.S.; PORTELA, L.A.P, \&MELO-SOUZA, S.E. de - Aneurismas dissecantes intracranianos da circulação posterior - Arg. Neuro-Psiquiat. 50: 351-360, 1992.

8. DUBARO, T.; POLICHOT, J.; LAMY, C.; HIER, D.; CAPLAN, L.R. \& WAS, J.C. - Upper limb peripheral motor deficits due to extracranial vertebral artery dissection. Cerebrovasc Dis 4: 88-91, 1994.

9. FISHER, C.M.; OJEMANN, R.G.; ROBENSON, G.H. Spontaneous dissection of cervicocerebral arteries. Can j Neurol Sci. (1978) 5: 9-19, 1978

10. GARCIA-MONGO, J.C.; CANTO, G.F. \& BELDARRAIN, M.G. - Bilateral vertebral artery dissection in a patient with afribinogemia, Stroke, 27: 2325-2327, 1996.

11. GOULD, D.B. \& CUNNINGHAN, K. - Internal carotid artery dissection after remote surgery. latrogenic complication of anesthesia. Stroke, 25: 1276-1278, 1994.

12. HART, R.G. \& EASTON, J.D. - Dissections of cervical and cerebral arteries - Neurologic Clinics. 1: 161-1 165, 1994.

13. HOSOYA, T.; WATANABE, N.; YAMAGUCHI, K.; KUBOTA, H. \& ONODERA, Y. - Intracranial vertebral artery dissection in Wallenberg syndrome. AJNR Am J Neuroradiol. 15: 1 - 161. 165,1994

14. KITANAKA, C.; SASAKI, T.; EGUCHI, T.; TERAOKA, A.; NAKANE, M. \& HOYA, K. - Intracranial vertebral artery dissections: clinical, radiological, features and surgical considerations. Neurosurgery. 34: 620-626, 1994.

15. KITANAKA, C.; TANAKA, J; KUWAHARA, M. \& TERAOKA, A - Magnetic resonance imaging study of intracranial vertebrovasilar artery dissections. Sstroke. 25: 571-575, 1994.

16. KUMRAL, E.; BOUGOUSSLAVSKY, J., VAN MELLE, G.; REGLI, F. \& PIERRE, P. - Headache at stroke onset: the Lausanne Stroke Registry. J Neurol Neurosurg Paychiatr. 58: 490-492, 1995

17. LARSON, T.C. 3rd, KELLY W.M.; EHMAN, R.L. \& WEHRLI, F.W. - Spatial misregistration of vascular flow during MR imaging of the CNS: cause and clinical significance. Amer $J$ Neuroradiol. 11: 1041-1048, 1990.

18. LECLERC, $X . ;$ GODEFROY, O.; SALHI, A.; LUCAS, C.; LEYS D. \& PRUND, J.P. - Helical CT for he diagnosis of extracranial internal carotid artery dissection. Stroke, 27: 461-466, 1996.

19 - LEVY, C.; LAISSY, J.P.; RAVEAN, V.; AMARENCO, P.; SERVOIR, V.; BOUSSER, MG. \& TUBIANA, J.M. - Carotid and vertebral artery dissections: 3 - dimensional time-of flight MR angiography and MR imaging versus conventional angiography - Radiology, 190: 97-103, 1993.

20. LEYS, D.; MOULIN, Th.; STOJKOVIC, T.; GEGEY, S. \& CHAVOT, D. (DONALD investigators) - Follow-up of patients with history of cervical artery dissection- Cerebrovasc Dis. 5: 43-49, 1995.

21. MAYER, S.A.; RUBIN, B.S.; STARMAN, B.J. \& BYENS, P.H. Spontaneous multiversal cervical artery dissection in a patient with a substitution of alamine for glycine (G13A) in the alfa! (I) chain of type I collagen - Neurology, 47: 552-556, 1996.

22. MOKRI, B. - Spontaneous dissections of cervococephalic arteries. In: Primer on Cerebrovascular Diseases, editado por K.M.A. Welch, Louis R. Caplan, Donald J. Reis, Bo K. Siesjo \& Bryce Weir, Academic Press, 1997, páginas 390-108.

23. NYGUEN BUI, L., BRANT-SAWADSKI, M., VERGHESE, P. \& GILLAN, G. - Magnetic ressonance angiography of cervicocranial dissection. Stroke, 24: 126-131, 1993.

24. POZZATI, E., GIULIANI G., ACCIARRI, N. \& NUZZO, G. Long-term follow-up of occlusive cervical carotid dissection. Stroke; 21: 528-531, 1990.

25. PROVENZALE, T.M. - Dissection of the internal carotid and vertebral arteries: imaging features. AJR Am J Roentgenol. 165: 1099-1104, 1995

26. SCHIEVINK, W.I., MOKRI, B. \& O'FALLON, W.N. • Recurrent spontaneous cervical-artery dissection. $n$ Engl J Med. 330: 3930397, 1994. 
27. SCHIEVINK, W.I.; MOKRI, B. \& WHISNANT, J.P. - Internal carotid artery dissection in a community. Rochester, Minessota, 1987-1992. Stroke, 24: 1678-1680, 1993.

28. SCHIEINK, W.F.; MOKRI, B \& PIEPGRASS, D.A. Spontaneous dissections of cervico-cephalic arteries in childhood and adolescence - Neurology, 44: 1607-1612, 1994.

29. SCHIEVINK, W.I.; PRAKASH, U.B.; PIEPGRASS, D.G. \& MOKRI, B. - Alpha I- antitrypsina deficiency in intracranial aneurism and cervical artery dissection. Lancet, 343: 452-453, 1994.

30. SILBERT, P.L.; MOKRI, B. \& SCHIEVINK, W.I. - Headache and neck brain in spontaneous internal carotid and vertebral artery dissections - Neurology 45: 1517-1522, 1995.

31. SRINIVASAN, J.; NEWELL, D.N.; STURZENEGGER, M; MAXBERG, M.R. WINN, H.R. - Transcranial doppler in the evaluation of internal carotid artery dissection. Stroke 27: 1226-1230, 1996.

32. STEINKE, W.; RAUTENBERG, W.; SCHWANTZ, A. \& HENNERICI, M. - Noninvasive monitoring of internal carotid artery dissection. Stroke, 25: 998-005, 1994. 33a. STURZENNEGER, M. - Headache and neck pain: The warming symptoms of vertebral artery dissection. headache 34: 187-193, 1994.

33b. STURZENEGGER, M. - Spontaneous internal carotid artery dissection: early diagnosis and management in 44 patients. J. Neurol. (Germany). 242: 231-38, 1995.

34. STURZENEGGER, M.; MATTLE, H.P.; RIVOIR, A., RIHS, F \& SCHMID, C. - Ultrasound findings in spontaneous extracranial vertebral artery dissection. Stroke, 24: 1910-1921, 1993.

35. SUE, D.E.; BRANT-ZAWADZKI, M.N. \& CHANCE, J. * Dissection of cranial arteries in the neck: correlation of MRI and arteriography. Neuroradiology, 34: 273-278, 1992.

36. TSUKAHARA, T.; WADA, H.; SATAKE, $K$; YAOITA, H. \& TAKAHASHI, A. - Proximal ballon occlusion for dissecting vertebral aneurysms accompanied by subarachnoid hemorrhage. Neurosurgery, 36: 914-919, 1995.

37. YOSHIMOTO, Y, \& WAKAI, S. - Unrupted intracranial vertebral artery dissection: clinical course and serial radiographic imagings. Stroke, 28: 370-374, 1997. 